\title{
Serial multidetector computed tomography assessment of left ventricular reverse remodeling, mass, and regional wall stress after restrictive mitral annuloplasty in dilated cardiomyopathy
}

\author{
Yasuhiro Shudo, MD, ${ }^{\mathrm{a}}$ Kazuhiro Taniguchi, MD, PhD, ${ }^{\mathrm{b}}$ Koji Takeda, MD, PhD, ${ }^{\mathrm{a}}$ Taichi Sakaguchi, MD, PhD, ${ }^{\mathrm{a}}$ \\ Toshihiro Funatsu, MD, PhD, ${ }^{\mathrm{b}}$ Haruhiko Kondoh, MD, PhD, ${ }^{\mathrm{b}}$ and Yoshiki Sawa, MD, PhD ${ }^{\mathrm{a}}$
}

Objective: To evaluate serial data on left ventricular and myocardial reverse remodeling after restrictive mitral annuloplasty.

\begin{abstract}
Methods: Thirteen patients (age, $64 \pm 6$ years) with functional mitral regurgitation associated with dilated cardiomyopathy (ejection fraction, $\leq 35 \%$ ) were examined before (baseline), soon ( $1.7 \pm 1.5$ months), and more than 1 year (16 \pm 8 months) after restrictive mitral annuloplasty using multidetector computed tomography. The left ventricular end-diastolic and end-systolic volume indexes, left ventricular ejection fraction, left ventricular mass index, and end-systolic wall stress were estimated.
\end{abstract}

Results: In the period soon after restrictive mitral annuloplasty, significant reverse left ventricular remodeling was present, with decreases in the left ventricular end-diastolic volume index $\left(149 \pm 42\right.$ to $119 \pm 41 \mathrm{~mL} / \mathrm{m}^{2}$, $P=.04)$ and left ventricular end-systolic volume index $\left(114 \pm 44\right.$ to $\left.78 \pm 43 \mathrm{~mL} / \mathrm{m}^{2}, P=.02\right)$, and an increase in left ventricular ejection fraction $(25 \% \pm 10 \%$ to $37 \% \pm 14 \%, P=.01)$. Additional changes in these parameters were seen in the later period $\left(103 \pm 29 \mathrm{~mL} / \mathrm{m}^{2}, 61 \pm 23 \mathrm{~mL} / \mathrm{m}^{2}\right.$, and $42 \% \pm 9 \%$, respectively; all $P<.05$ vs baseline). In the early postrestrictive mitral annuloplasty period, the left ventricular mass index did not change significantly (104 \pm 22 to $\left.104 \pm 18 \mathrm{~g} / \mathrm{m}^{2}, P=\mathrm{NS}\right)$, but significant regression occurred in the later period ( $90 \pm 17 \mathrm{~g} / \mathrm{m}^{2}, P<.05$ vs baseline). The end-systolic wall stress was significantly decreased in the early period $(P<.05)$ and was sustained in the late period. Furthermore, the increase in left ventricular ejection fraction in the late period correlated significantly with the magnitude of the end-systolic wall stress reduction $(r=-0.67, P=.01)$.

Conclusions: Our findings indicate that ventricular reverse remodeling occurs soon after restrictive mitral annuloplasty. In contrast, myocardial reverse remodeling (ie, regression of myocardial hypertrophy) occurs over time between the early and late postoperative periods. Our data also suggest that the late improvement in left ventricular systolic performance might be attributable to a decrease in the left ventricular afterload. (J Thorac Cardiovasc Surg 2012;143:S43-7)

Functional mitral regurgitation (MR) is a common complication of both ischemic and nonischemic dilated cardiomyopathy. MR leads to increased left ventricular (LV) volume overload, which exacerbates the LV remodeling process, eventually leading to congestive heart failure. ${ }^{1}$ To resolve

From the Department of Cardiovascular Surgery, ${ }^{\mathrm{a}}$ Osaka University Graduate School of Medicine, Suita, Osaka, Japan; and Department of Cardiovascular Surgery, ${ }^{\mathrm{b}}$ Japan Labor Health and Welfare Organization, Osaka Rosai Hospital, Sakai, Osaka, Japan.

This research was partially supported by funds to promote the hospital function of the Japan Labor Health and Welfare Organization.

Disclosures: Yasuhiro Shudo, Kazuhiro Taniguchi, Koji Takeda, Taichi Sakaguchi, Toshihiro Funatsu, Haruhiko Kondoh, and Yoshiki Sawa have nothing to disclose with regard to commercial support.

Presented at The American Association for Thoracic Surgery Mitral Conclave, New York, New York, May 5-6, 2011.

Received for publication April 30, 2011; revisions received Oct 3, 2011; accepted for publication Nov 8, 2011; available ahead of print Dec 12, 2011.

Address reprints to Yoshiki Sawa, MD, PhD, Department of Cardiovascular Surgery, Osaka University Graduate School of Medicine, Osaka, Japan (E-mail: sawa-p@ surg 1.med.osaka-u.ac.jp).

$0022-5223 / \$ 36.00$

Copyright (c) 2012 by The American Association for Thoracic Surgery

doi:10.1016/j.jtcvs.2011.11.013 this vicious circle, Bolling and colleagues ${ }^{2}$ reported that restrictive mitral annuloplasty (RMA) is feasible in patients with end-stage cardiomyopathy. Since then, short-term and midterm survival rates and the extent of ventricular reverse remodeling (decrease in LV volume) after MR correction have been reported. ${ }^{2,3}$ However, follow-up data on ventricular reverse remodeling after RMA in patients with end-stage cardiomyopathy are scarce. Moreover, the existing follow-up studies used echocardiography, which is not optimal for a precise assessment.

Multidetector computed tomography (MDCT) is an emerging technique that enables more accurate contour definition than echocardiography. With electrocardiogramgated image acquisition, cardiac MDCT shows different planes of the whole heart and enables the assessment of 3-dimensional volume and ejection performance. We recently developed MDCT-based analysis software to compute local circumferential myocardial stress. ${ }^{3-5}$

The objective of the present study was to assess the serial changes in the LV volumes, function, and mass and in regional myocardial stress before, and early and late after 


$$
\begin{aligned}
& \text { Abbreviations and Acronyms } \\
& \text { DCM = dilated cardiomyopathy } \\
& \text { ESS = end-systolic wall stress } \\
& \text { LVEF = left ventricular ejection fraction } \\
& \text { LVEDVI = left ventricular end-diastolic volume } \\
& \text { index } \\
& \text { LVESVI }=\text { left ventricular end-systolic volume } \\
& \text { index } \\
& \text { LVMI = left ventricular mass index } \\
& \text { MDCT = multidetector computed tomography } \\
& \text { MR = mitral regurgitation } \\
& \text { MRI = magnetic resonance imaging } \\
& \text { RMA = restrictive mitral annuloplasty }
\end{aligned}
$$

RMA in patients with nonischemic dilated cardiomyopathy (DCM) and functional MR, using cine-MDCT and the software we developed.

\section{METHODS \\ Patients}

From January 2007 to December 2008, 24 patients with functional MR (grade $3+$ or greater, regurgitant volume $>30 \mathrm{~mL} / \mathrm{beat}$ ) associated with DCM were referred for surgery. Of these patients, 13, who underwent serial cine-MDCT analysis, were included in the present study. All had congestive heart failure symptoms, a history of at least 1 hospitalization, and advanced LV remodeling, as defined by an LV ejection fraction (LVEF) of $\leq 0.35$ and an end-systolic volume $>120 \mathrm{~mL}$, on preoperative left ventriculography. Patients with organic MR, a rheumatic mitral valve, or aortic valve disease were excluded. The patient characteristics are shown in the Table 1. All patients were receiving optimized medical regimens, including $\beta$-blockers, angiotensin-converting enzyme inhibitors or angiotensinreceptor blockers, and diuretics.

Our institutional ethics committee approved the study, and all patients provided written informed consent for all procedures before surgery.

\section{Surgical Procedures}

Surgery was performed through a median sternotomy under mild hypothermic cardiopulmonary bypass with antegrade and retrograde intermittent cold blood cardioplegia. All patients underwent a stringent RMA (2-4 ring sizes smaller than measured; Table 1) with a semirigid ring (Carpentier-Edwards Physio Ring; Edwards LifeSciences, Irvine, Calif). Postoperative MR was none or trivial in all patients.

\section{Cine-MDCT Angiography}

Electrocardiogram-gated MDCT examinations of the heart were performed 1 month before (baseline) and again early ( $1.7 \pm 1.5$ months) and late (16 \pm 8 months) after RMA using a commercially available 64-slice multidetector scanner (Somatom Definition Dual Source CT, Siemens, Germany). To enhance the $\mathrm{LV}$ cavity, $0.8 \mathrm{~mL} / \mathrm{kg}$ of nonionic-contrast medium was administered to the patient at $4 \mathrm{~mL} / \mathrm{s}$. The scanning delay was set with an automatic triggering system. To ensure that the contrast material in the left ventricle was at its maximum concentration, a circular region of interest for density measurement was defined in the ascending aorta. As soon as the signal intensity in the region of interest reached a threshold of 150 Hounsfield units, the patient was instructed to maintain an end-inspiratory breath-hold, and data acquisition was started. Computed tomography was performed with a tube voltage of $120 \mathrm{kV}$, effective tube current-time product of $180 \mathrm{~mA}$, collimation of $64 \times 0.625 \mathrm{~mm}$, beam pitch of 0.23 , tube speed of $9.2 \mathrm{~mm} /$ rotation, gantry rotation time of $350 \mathrm{~ms}$, and estimated radiation dose of $18 \mathrm{mSv}$. The computed tomography data of the entire heart were reconstructed using retrospective electrocardiogram-gating and a standard cardiac algorithm. From early systole to late diastole, 10- or 20 -image series were reconstructed at $5 \%$ or $10 \%$ R-R interval increments throughout the cardiac cycle $(5 \%-95 \%)$. Routine LV function variables, such as the LV end-diastolic volume, LV end-systolic volume, LVEF, and LV mass, were measured and calculated according to the slice summation method on a workstation, using LV functional analysis software (Aquarius Net Station, version 1.5; Terarecon, San Mateo, Calif). The volumes and mass were indexed to the body surface area. All image processing was verified by experienced radiologists (M.K. and S.H.).

\section{Assessment of Regional Myocardial Wall Stress}

The imaging analysis used to assess regional myocardial stress was performed on a personal computer with dedicated analysis software (Osaka University-OSCAR STRESS tool, Osaka, Japan), as described previously. ${ }^{3-5}$ The regional end-systolic stress (ESS) was computed at $16 \mathrm{seg}-$ ments (ie, excluding the apical cap; segment no. 17). The regional circumferential stresses were also averaged at $3 \mathrm{LV}$ levels (base, mid-LV, and apex).

\section{Statistical Analysis}

SPSS, version 16.0 (SPSS, Chicago, Ill) software was used for statistical analyses. Continuous values are expressed as the mean \pm standard deviation or the mean value. Normally distributed variables were compared using Student's $t$ test for paired or unpaired data. Repeated measures analysis of variance was used to compare the mean parameters obtained at different points. The significance of the differences was determined using a 2-tailed multiple $t$ test with Bonferroni's correction. Correlations between variables were tested with Pearson's correlation analysis. $P<.05$ was considered statistically significant.

\section{RESULTS \\ Clinical Results}

All patients tolerated the operation well. The mean New York Heart Association functional class had improved significantly, from $2.9 \pm 0.5$ to $1.5 \pm 0.5(P<.001)$ soon after surgery, and remained unchanged at the late assessment $(P<.001$ vs before surgery, $P=\mathrm{NS}$ vs soon after surgery). The degree of MR improved significantly, from $3.5 \pm 0.5$ to $0.6 \pm 0.8(P<.0001)$, soon after surgery and remained unchanged at the late postoperative point $(P<.0001$ vs before surgery, $P=\mathrm{NS}$ vs soon after surgery). During the followup period after discharge, no patients developed heart failure or MR recurrence.

\section{Serial Change in LV Volumes}

In the early period after RMA, significant reverse LV remodeling occurred, with decreases in the left ventricular end-diastolic volume index (LVEDVI; $149 \pm 42$ to $119 \pm$ $\left.41 \mathrm{~mL} / \mathrm{m}^{2}, P=.04\right)$ and left ventricular end-systolic volume index (LVESVI; $114 \pm 44$ to $78 \pm 43 \mathrm{~mL} / \mathrm{m}^{2}, P=.02$ ). Additional reverse remodeling changes were seen late after surgery $\left(103 \pm 29 \mathrm{~mL} / \mathrm{m}^{2}\right.$ and $61 \pm 23 \mathrm{~mL} / \mathrm{m}^{2}$, respectively; all $P<.05$ vs baseline; Figure 1). Although no significant difference was found in LDEDVI or LVESVI between the early 
TABLE 1. Patient characteristics

\begin{tabular}{lc}
\hline \multicolumn{1}{c}{ Variable } & $\mathbf{n}=\mathbf{1 3}$ \\
\hline Patient characteristics & \\
Age (y) & $64 \pm 6$ \\
Male (n) & 12 \\
Body surface area $\left(\mathrm{m}^{2}\right)$ & $1.73 \pm 0.16$ \\
NYHA & $2.9 \pm 0.5$ \\
LVEF (\%) & $25 \pm 10$ \\
Surgical data & \\
RMA & 13 \\
Ring size used & 10 \\
$\quad$ Physio Annuloplasty Ring no. 24 (n) & 3 \\
$\quad$ Physio Annuloplasty Ring no. 26 (n) & 12 \\
Concomitant procedure & 5 \\
$\quad$ TAP (n) & \\
Maze (n) & $223 \pm 60$ \\
Intraoperative data & $125 \pm 44$ \\
$\quad$ CPB time (min) & \\
AXC time (min) & \\
\hline Data are presented as mean value \pm SD or number of patients. NYHA, New York Heart \\
Association; $L V E F$, left ventricular ejection fraction; $R M A$, restrictive mitral annulo- \\
plasty; TAP, tricuspid annuloplasty; $C P B$, cardiopulmonary bypass; $A X C$, aortic \\
crossclamp.
\end{tabular}

and late postoperative assessments $(P=.31$ and $P=.27$, respectively), the average percentage of reduction in the mean LVEDVI (vs baseline) was 20\% soon after surgery and $31 \%$ later after surgery; however, for the LVESVI, it was 32\% soon after surgery and $46 \%$ in the late postoperative period.

\section{Serial Change in Global Systolic Performance}

The LVEF increased significantly in the early post-RMA period $(25 \% \pm 10 \%$ to $37 \% \pm 14 \%, P=.01)$, with additional improvement by the late postoperative period $(42 \%$ $\pm 9 \%, P<.05$ vs baseline; Figure 1$)$. However, no significant difference was found in the LVEF between the early and late periods after surgery $(P=.29)$, the average postoperative percentage of improvement in the LVEF (vs baseline) was $48 \%$ at the early postoperative point and $68 \%$ at the late follow-up period.

\section{Serial Change in LV Mass}

In the early postoperative period, the LV mass index (LVMI) did not change significantly $(104 \pm 22$ to $104 \pm$ $\left.18 \mathrm{~g} / \mathrm{m}^{2}, P=\mathrm{NS}\right)$, but significant regression was observed in the late period $\left(90 \pm 17 \mathrm{~g} / \mathrm{m}^{2}, P<.05\right.$ vs baseline; Figure 1). However, no significant difference was found in the LVMI between the early and late periods after surgery $(P=.11)$. The average postoperative percentage of reduction (vs baseline) in LVMI was $0 \%$ in the early stage and $13 \%$ at the late stage.

\section{Serial Change in Regional End-Systolic Wall Stress}

Regional ESS was significantly decreased in all 3 regions (base, midleft ventricle, and apex) in the early period
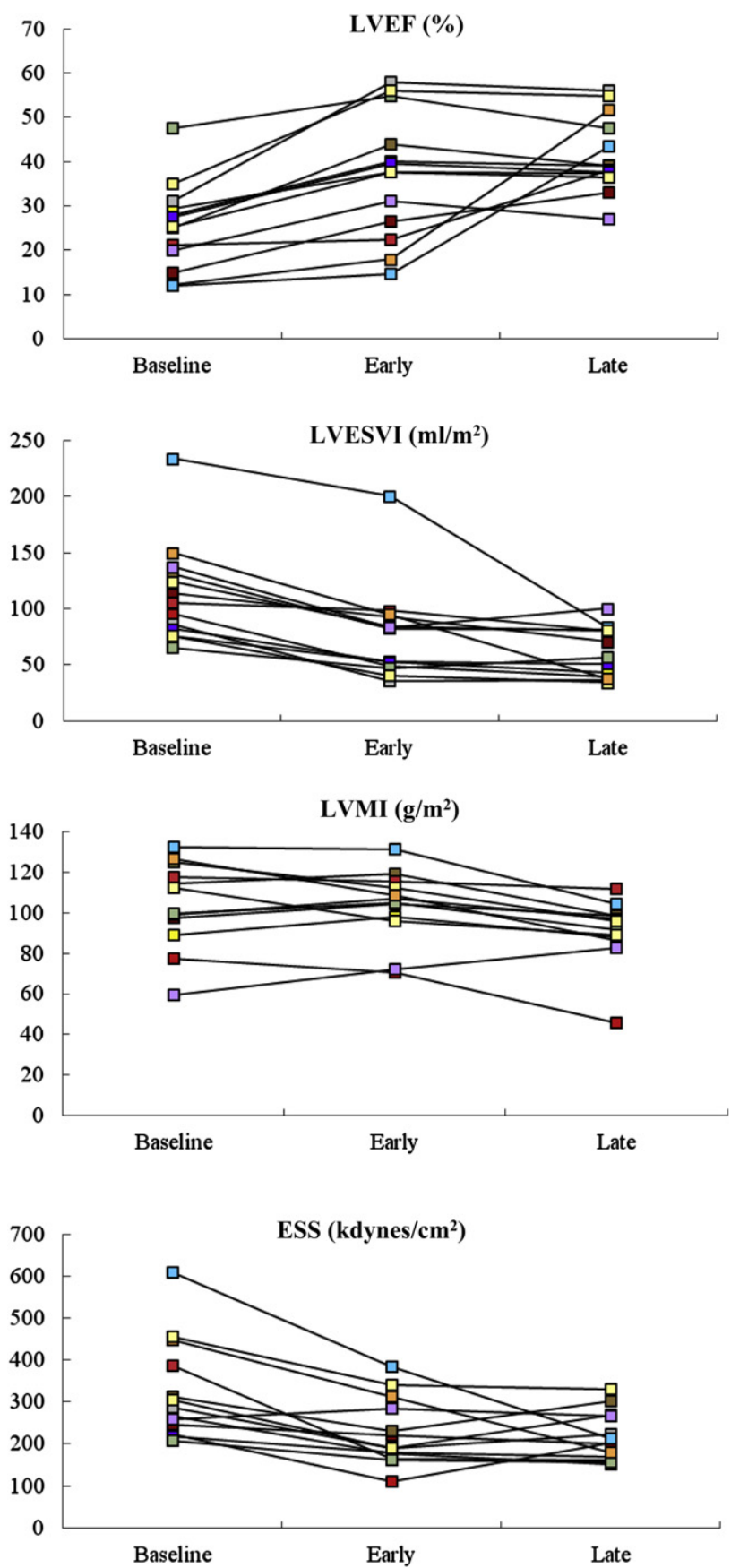

FIGURE 1. Serial changes in left ventricular ejection fraction $(L V E F)$, left ventricular end-systolic volume index (LVESVI), left ventricular mass index (LVMI), and end-systolic wall stress (ESS). Examinations were performed before (baseline) and early (at $1.7 \pm 1.5$ months) and late (at $16 \pm 8$ months) after surgery. Different symbols represent individual patients.

$\left(333 \pm 96\right.$ to $234 \pm 68 \mathrm{kdynes} / \mathrm{cm}^{2}, 345 \pm 138$ to $249 \pm$ $94 \mathrm{kdynes} / \mathrm{cm}^{2}, 288 \pm 133$ to $184 \pm 85 \mathrm{kdynes} / \mathrm{cm}^{2}$, respectively, all $P<.05$ ), and the decrease was well sustained at the late measurement $\left(219 \pm 55 \mathrm{kdynes} / \mathrm{cm}^{2}, 238 \pm 66\right.$ $\mathrm{kdynes} / \mathrm{cm}^{2}$, and $185 \pm 60 \mathrm{kdynes} / \mathrm{cm}^{2}$, respectively; 
Figure 1). No significant difference was found in the LVMI between the early and late periods after surgery $(P=.61$, $P=.80$, and $P=.98$, respectively). In the early postoperative period, the average percentage of reduction (vs baseline) in regional ESS was 30\% in the base, $28 \%$ in the midleft ventricle, and $36 \%$ in the apex. In the late period, it was $34 \%$ in the base, $31 \%$ in the midleft ventricle, and $36 \%$ in the apex.

\section{Relationship Between Regional End-Systolic Stress and Global Systolic Performance}

The increase in the LVEF observed in the late postoperative period correlated significantly with the magnitude of reduction in the mean regional ESS value in the 3 regions $(r=-0.67, P=.01)$.

\section{Relationship Between New York Heart Association Functional Class and LV Reverse Remodeling}

No significant correlation was found between the change in the New York Heart Association functional class and the change in the parameters related to LV reverse remodeling at either the early $(r=0.04, r=0.29$, and $r=0.08$ for ESS, LVESVI, and LVEF, respectively; $P=\mathrm{NS}$ for all) or late ( $r=0.26, r=0.22$, and $r=0.42$ for ESS, LVESVI, and LVEF, respectively; $P=\mathrm{NS}$ for all) postoperative assessment.

\section{DISCUSSION}

The major findings of the present study are that, after RMA, (1) the LVEDVI and LVESVI decreased significantly over time; (2) the LVEF significantly increased over time; (3) the LVMI did not change in the early postoperative period, but had significantly decreased by the late period; (4) the regional ESS significantly decreased early after surgery and was well maintained late after surgery; and (5) the increase in LVEF in the late postoperative period correlated significantly with the magnitude of reduction in the mean regional ESS value. Our findings indicate that ventricular reverse remodeling occurs soon after the correction of functional MR but that myocardial reverse remodeling (ie, the regression of myocardial hypertrophy) is a process that occurs over time, between the early and late postoperative periods. Our data also suggest that the late improvement in LV systolic performance might be attributable to a decrease in LV afterload.

The mechanism and process of the reverse remodeling have not been fully evaluated. ${ }^{3}$ The present study focused on the serial changes in a detailed fashion on various aspects of the reverse remodeling process, such as the LV volume, LV functional performance, regional wall stress, and LV mass, as measured using MDCT images, in patients with functional MR associated with DCM who underwent RMA. We must note that in previous reports LV reverse remodeling (eg, LV volumes and mass) was noted in early
( 2 months) and was present in long (3-4 years) follow-up after RMA in the patients with DCM. ${ }^{6}$ They showed that in 22 patients with DCM undergoing RMA, the mean LVEDVI and LVESVI had decreased from 110 to $80 \mathrm{~mL} / \mathrm{m}^{2}$ and from 42 to $31 \mathrm{~mL} / \mathrm{m}^{2}$ at 43 months after surgery. ${ }^{6}$ In contrast to those serial studies, our study enrolled patients with a more severely dilated left ventricle (LVEDVI, $149 \mathrm{~mL} / \mathrm{m}^{2}$; LVESVI, $114 \mathrm{~mL} / \mathrm{m}^{2}$ ). On the basis of our results that ventricular reverse remodeling occurred earlier than myocardial reverse remodeling, we have attempted to explain the time discrepancy between the ventricular and myocardial reverse remodeling. We speculate that the release from volume overload and the subsequent reduction in wall stress might initiate a gradual response that results in the regression of myocardial hypertrophy. Furthermore, our data, together with the concept of Grossman and colleagues ${ }^{7}$ of volumerelated cell thickening and elongation (ie, hypertrophy), suggest that abolishing volume overload might activate cellular and extracellular mechanisms that modify myocardial structural remodeling. Among the many complex processes associated with reverse remodeling, eliminating MR can cause changes in the ventricular geometry and the subsequent adjustable response in the molecular signals that promote hypertrophy and extracellular matrix turnover. ${ }^{8}$ Taken together with previous studies, our data support a possible mechanism that ventricular reverse remodeling is followed by myocardial reverse remodeling.

The serial changes (acute and chronic) in myocardial stress after RMA have not been fully investigated, because it can be difficult to measure regional myocardial stress in left ventricles with different shapes. In the present study, we sought to assess the serial changes in regional myocardial stress after RMA in patients with functional MR. We observed that the late improvement in LV systolic performance correlated well with a decrease in LV afterload. Our data suggest that the regional dysfunction of the residual myocardium might be reversible, at least in part, although whether the improvement is related to the reduction in myocardial wall stress or to an alteration in the myocardial contractile property itself still needs to be evaluated.

Echocardiography has been widely used to measure the LV volume and mass in heart failure, but the results were based on geometric assumptions that limit the accuracy of the data. Currently, cardiac magnetic resonance imaging (MRI) is considered the reference standard for LV geometry, volume, and mass evaluation. ${ }^{6}$ It provides excellent temporal and spatial resolution, with a high degree of accuracy and reproducible quantitative measurements. The multiphase cine imaging quality is significantly better in MRI than in MDCT, owing to the differences in temporal resolution. However, MRI requires an extended scanning period and cannot be used for patients with a pacemaker. Previous computed tomography studies have shown a good correlation and acceptable agreement of LV volumes and cardiac 
function with those obtained by MRI. ${ }^{9}$ This is probably because the LV volumes and cardiac function are mainly determined by the end-systolic and end-diastolic phases, when cardiac motion is comparatively minimal and motion artifacts are negligible. Therefore, 64-row MDCT appears to be a useful alternative to 2-dimensional or 3-dimensional echocardiography or MRI in study populations with metal implants (eg, pacemakers or implantable cardioversiondefibrillators), claustrophobia, or other conditions that contraindicate MRI.

\section{Clinical Implications}

The present results have shown that in patients undergoing RMA who have DCM and functional MR, a better understanding of the ventricular response to surgical interventions could be very helpful in selecting favorable candidates or determining the timing of surgery.

\section{Study Limitations}

The major limitations of our study were that it was not a randomized study, and it involved a rather small sample size. The study would have been stronger with a larger, randomized, and controlled population.

Regarding the technical aspects, the variety of surgical procedures (eg, tricuspid annuloplasty and Maze) and type of annuloplasty ring could have influenced the outcomes. However, these concomitant procedures are usually performed in very sick populations with similar clinical and pathologic findings to those of our patients, regardless of the etiology of the LV dysfunction.

We performed RMA with the implantation of an undersized semirigid ring. In 1 patient with a small body surface area $\left(1.55 \mathrm{~m}^{2}\right)$, we used a ring undersized by 4 ring sizes, as indicated when the annular diameter exceeds $34 \mathrm{~mm}$. However, the use of such a small ring might result in functional mitral stenosis after the RMA. ${ }^{10}$ In the present study, we did not evaluate the serial change in pulmonary hypertension and/or right ventricular function.

All the patients enrolled in the present study had DCM; therefore, our results are not applicable to patients with ischemic DCM.
As with conventional modalities, MDCT has inherent limitations, including the need for contrast medium and radiation exposure.

\section{CONCLUSIONS}

Our MDCT findings indicate that ventricular reverse remodeling occurs soon after RMA, although myocardial reverse remodeling (ie, the regression of myocardial hypertrophy) is a process that occurs over time between the early and late postoperative periods.

The authors thank Dr Seiki Hamada of Radiology, Osaka University Graduate School of Medicine and Mr. Koji Kubo of Radiology, Osaka Rosai Hospital, for data acquisition.

\section{References}

1. Mann DL. Mechanisms and models in heart failure: a combinatorial approach. Circulation. 1999;109:999-1008.

2. Bolling SF, Pagani FD, Deeb GM, Bach DS. Intermediate-term outcome of mitral reconstruction in cardiomyopathy. J Thorac Cardiovasc Surg. 1998;115 381-6.

3. Takeda K, Taniguchi K, Shudo Y, Kainuma S, Hamada S, Matsue H, et al. Mechanism of beneficial effects of restrictive mitral annuloplasty in patients with dilated cardiomyopathy and functional mitral annuloplasty. Circulation. 2010;122 S3-9.

4. Shudo Y, Taniguchi K, Takeda K, Sakaguchi T, Matsue H, Izutani H, et al. Assessment of regional myocardial wall stress before and after surgical correction of functional ischaemic mitral regurgitation using multidetector computed tomography and novel software system. Eur J Cardiothorac Surg. 2010;38: 163-70.

5. Shudo Y, Matsumiya G, Takeda K, Matsue H, Taniguchi K, Sawa Y. Novel software package for quantifying local circumferential myocardial stress. Int J Cardiol. 2011;147:134-6.

6. Westenberg JJ, Braun J, Van de Veire NR, Klautz RJ, Versteegh MI, Roes SD, et al. Magnetic resonance imaging assessment of reverse left ventricular remodeling late after restrictive mitral annuloplasty in early stages of dilated cardiomyopathy. J Thorac Cardiovasc Surg. 2008;135:1247-53.

7. Grossman W, Jones D, McLaurin L. Wall stress and patterns of hypertrophy in the human left ventricle. J Clin Invest. 1975;56:56-64.

8. Beeri R, Yosefy C, Guerrero JL, Nesta F, Abedat S, Chaput M, et al. Mitral regurgitation augments post-myocardial infarction remodeling failure of hypertrophic compensation. J Am Coll Cardiol. 2008;51:476-86.

9. Reman SV, Shar M, McCarthy B, Garcia A, Ferketich AK. Multi-detector row cardiac computed tomography accurately quantifies right and left ventricular size and function compared with cardiac magnetic resonance. Am Heart J. 2006;151:736-44.

10. Kainuma S, Taniguchi K, Daimon T, Sakaguchi T, Funatsu T, Kondoh H, et al Does stringent restrictive annuloplasty for functional mitral regurgitation cause functional mitral stenosis and pulmonary hypertension? Circulation. 2011;75: 538-9. 